\title{
Stage IIIC Fallopian Tube Cancer AJCC v7
}

National Cancer Institute

\section{Source}

National Cancer Institute. Stage IIIC Fallopian Tube Cancer AJCC v7. NCI Thesaurus. Code C6275.

Stage IIIC includes: (T3c, N0, M0); (Any T, N1, M0). T3c: Peritoneal metastasis outside the pelvis and more than $2 \mathrm{~cm}$ in diameter. N0: No regional lymph node metastasis. N1: Regional lymph node metastasis. M0: No distant metastasis. (AJCC 7th ed.) 